\title{
El carácter ambiguo de la fusión de ho- rizontes en la hermenéutica filosófica de Hans-Georg Gadamer
}

\section{The ambiguity of horizons fusion in Hans-Georg Gadamer's philosophic hermeneutics}

\author{
LEANDRO CATOGGIO
}

CONICET y Universidad Nacional del Mar del Plata (Argentina)

Recibido: 21-03-2008 Aprobado definitivamente: 05-11-2008

\section{RESUMEN}

rtir de las problemáticas desarrolladas por Charles Taylor y su explícita adhesión al programa imeriano de la hermenéutica se intentará desarrollar la noción de fusión de horizontes con ecto a dos cuestiones inherentes a ella. Por un lado el papel que cumple en el entendimiento e esquemas comprensivos diferentes; y por otro lado, la ambigüedad del carácter de la misma 6n. Esto en referencia a lo siguiente: o bien se trata de la fusión de horizontes distintos o se trata de una fusión interna en un solo horizonte, con lo cual lo primero resulta ser sólo mera apariencia.

\section{PALABRAS CLAVE \\ HERMENÉUTICA, GADAMER, FUSIÓN DE HORIZONTES, ALTERIDAD}

\section{ABSTRACT}

is paper I analyse Charles Taylor's notion of horizons fusion, which comes from Gadamer's meneutics (as Taylor avows). Two problems are discussed: on the one hand, the role of this on regarding the understanding between different comprehensive schemes and, on the other the ambiguity of fusion in itself, insofar as it can be seen either as the fusion of different zons, or as an internal fusion in the context of one horizon. Thus the first possibility becoa mere appearance. 


\section{INTRODUCCIÓN}

Charles Taylor menciona en Su texto «Comparison, History, Truth» cuatro discusiones interdisciplinarias que comprenden la problemática del entendimiento intersubjetivo entre esquemas conceptuales diferentes. ${ }^{1}$ Cuestiones éstas que se presentan en la configuración actual de las ciencias sociales. Ellas, sucintamente, son las siguientes. La primera refiere a la difusa frontera entre mito y religión así como la segunda al papel que juega tanto una religión como una sociedad en una conceptuación determinada de la historia. La tercera implica aquello en lo que nos detendremos en referencia a la hermenéutica gadameriana: la posibilidad de comprensión de una cultura ajena sin caer en un etnocentrismo. La cuarta, en íntima conexión con la anterior, señala la necesariedad o no del juzgamiento de una cultura extraña en nuestro intento de comprenderla. Estas cuestiones que releva Taylor al comienzo de su escrito apuntan a desarrollar el entendimiento intercultural desde la noción de «comparación». Dicho concepto plantea la posibilidad de la comprensión desde un contraste (contrast) entre el esquema discursivo propio y el esquema discursivo ajeno. Esta comparación tiene la virtud de, por un lado, no caer en un etnocentrismo y, por otro lado, de crear un lenguaje en común de comprensión mutua. Es esto mismo lo que lleva a Taylor a emparentarse con Gadamer diciendo que «el objetivo es la fusión de horizontes y no el escapar a los horizontes». ${ }^{2}$ Nosotros nos adentraremos en la propuesta gadameriana de la «fusión de horizontes» (Horizontverschmelzung) e intentaremos ver no sólo cómo es su funcionalidad dentro de la hermenéutica filosófica sino también las problemáticas que ella misma plantea en su constitución. Esto nos llevará a tratar también los otros puntos planteados por Taylor siguiendo la argumentación gadameriana. Sobretodo ahondaremos en la relación esencial que tiene la fusión de horizontes con la noción de historia.

\section{LA FUSIÓN DE HORIZONTES}

Gadamer al reemplazar la noción de ser-para-la-muerte por la de ser-parael-texto reconduce el carácter de la comprensión a la unidad de sentido presente en el discurso. Siempre operamos en nuestra interpretación con el presupuesto de una unidad de sentido del texto. Sea éste hablado o escrito para Gadamer existe

1 Ch. Taylor, «Comparison, History, Truth» en Ch. Taylor, Philosophical Arguments, Cambridge-London, Harvard University Press, 1995, pp. 146-164 (trad. castellana Taylor, Ch. Argumentos filosóficos, traducción de Fina Birulés Bertrán, Barcelona, Paidós, 1997, pp. 199-220). De aquí en más se citará la versión original de la obra y la versión castellana entre paréntesis.

2 «The aim is fusion of horizons, not escaping horizons», Ibíd. p. 151 (p. 205). 
siempre un presupuesto formal que guía todo acto interpretativo, el anticipo de perfección (Vollkommenheit). ${ }^{3}$ Sin duda, aquí están operando las tres coordenadas de la interpretación que se pueden observar en la estructura comprensiva del Dasein que esboza Heidegger en Sein und Zeit. ${ }^{4} \mathrm{El}$ haber previo (Vorhabe) es resignificado por Gadamer en el anticipo de perfección y viene a considerar tanto una unidad de pre-comprensión en la que nos hallamos y que nos permite tener una manera previa de ver (Vorsicht) como una concepción previa (Vorgriff) con pretensión de verdad. De allí que el concepto de «tradición» (Überlieferung) en Gadamer tenga sentido ambiguo. Puede entenderse tanto como la simple transmisión de sentido a través del tiempo como también la transmisión de un contenido verdadero normativo. El punto, de todas maneras, radica en que la tarea de la hermenéutica no es la de desplegar un método de comprensión, sino clarificar las condiciones bajo las cuales se comprende. Esta tarea de averiguar las condiciones de comprensión nos remonta al esclarecimiento de los prejuicios (Vorurteile) inherentes al acto comprensivo. Lo esencial a la iluminación de los prejuicios determinantes implica que los mismos se nos ofrezcan, que nos sean dados. A nuestro entender, aquí es donde podemos diferenciar la postura gadameriana de la heideggeriana en torno al círculo hermenéutico. En Wahrheit und Methode se explicita que el conjunto de prejuicios que conforman la tradición poseen dos características fundamentales: son indistinguibles y, por ello mismo, indisponibles. Esto es, los prejuicios no están a disposición del intérprete de una manera accesible donde pueda descartar aquellos que sean productivos de aquellos que interfieran en la comprensión de la cosa.

No hay una accesibilidad plena al haber previo (Vorhabe) que nos pueda mostrar la génesis temporal del sentido. Justamente esta falta de claridad en la distinción entre prejuicios productivos e improductivos es lo que lleva a que los mismos se nos presenten como indisponibles. Existe una indisponibilidad esencial del lenguaje que hace que en la iluminación de las condiciones de comprensión siempre halla un resto indistinguible en el conjunto de prejuicios. El sentido no se agota ni en el autor ni en las condiciones contextuales de la obra; modo éste último de la hermenéutica romántica. El mismo posee posibilidades innumerables en su historicidad que son inabarcables para el carácter finito de nuestra comprensión. Por ello no puede haber ni una transparencia de sentido,

3 Cfr. H-G Gadamer, Wahrheit und Methode. Mohr, Tubinga, 1975, p 278. (trad. castellana Verdad y Método, traducción de Ana Aparicio-Rafael de Agapito, Salamanca, Sígueme, 1977, p 363.) De aquí en más se hará referencia a la obra original con las siglas WM seguida de la paginación y la traducción entre paréntesis con las siglas VM, seguida también de la paginación.

4 Cfr. M. Heidegger, Sein und Zeit, Max Niemayer, Tubinga, 1963, p. 232 (trad. castellana Ser y Tiempo, traducción por Jorge Rivera, Santiago de Chile, Editorial Universitaria, 1997, p 252). 
y por tanto una finalización de la tarea hermenéutica, ni una comprensión única del texto transmitido. La tarea hermenéutica es un proceso infinito y siempre se comprende de manera distinta, diferente. No podemos hablar de la validez de una única interpretación atemporal en la hermenéutica filosófica. La misma, debido a su propia estructura interna del acontecimiento, del suceder propio de la palabra, imposibilita una fijación expresa del sentido del texto. Tanto ello como la reconstrucción del contexto de la obra y el descubrimiento de la intención del autor plasmada en la obra resultan ajenos al planteamiento gadameriano. La conciencia histórica radica, justamente, en su carácter ontológico. Debido a ello es imposible desligarse completamente de los prejuicios latentes en la interpretación. El factor hermenéutico de la distancia temporal viene a considerar esa distancia insalvable de la interpretación y la mediación con el presente de la misma. La distancia en el tiempo (Zeitenabstandes) es la fusión de horizontes entre el presente y el pasado del sentido. Con ello obtenemos un precepto fundamental de la hermenéutica: el carácter temporal del sentido. Es el tiempo el fundamento del acontecer del mismo. Gadamer menciona al respecto lo siguiente: «El tiempo ya no es necesariamente un abismo que hubiera de ser salvado porque por sí mismo sería causa de división y lejanía, sino que es en realidad el fundamento que sustenta el acontecer en el que tiene sus raíces el presente». 5

Esta idea del tiempo donde tanto el presente y el pasado se hallan en mutua mediación se refleja, para Gadamer, en la llamada «regla hermenéutica» (hermeneutische Regel). ${ }^{6}$ Nos detendremos en ella para observar, justamente, cómo en la interpretación de textos la comprensión se presenta como un acontecimiento de la fusión de horizontes (Horizontverschmelzung) donde el sentido del texto se presenta como una tarea hermenéutica infinita. La regla hermenéutica, que Gadamer indica que es de origen retórico, representa la medida en que el todo configura la parte y, a su vez, la parte al todo. Ella es el continuo movimiento del lenguaje, del sentido para ser más precisos. La regla no es más que el modo de ser del acontecimiento del sentido. Es la enérgeia misma del sentido configurado por el tiempo. Para Gadamer esta idea de círculo es una unidad de constante reactualización en que lo individual reconfigura en cada nueva situación interpretativa el sentido de la obra. Esto es, el significado de la obra se reinterpreta con la mediación presente del horizonte del intérprete. La fusión de horizontes se hace efectiva en cada lectura del texto. Pero esto no implica una libertad sin límites en la interpretación. En ello no podemos reconocer una

5 «Nun ist die Zeit nicht mehr primär ein Abgrund, der überbrückt werden muss, weil er trennt und fernhät. Sondern sie ist in Wahrheit der tragende Grund des Geschehens, in dem das Gegenwärtige wurzelt» WM: 281 (VM: 367).

6 Cfr. WM: 275-276 (VM: 360-361). 
arbitrariedad del lector al modo de una construcción absoluta del significado. Lejos se encuentra Gadamer de la visión deconstructiva del posestructuralismo francés. No puede haber nunca una elaboración propia del interpretante. Esto implicaría no sólo la ceguera ante el horizonte de sentido del texto mismo sino también la ruptura del principio hermenéutico de la apertura, la vinculatividad moral inherente a toda comprensión. La vinculatividad se expresa en la resistencia a lo incomprensible y en el dejar valer contra mi propio horizonte la validez del horizonte confrontado en el texto. Esto es lo que entiende Gadamer por la forma de la experiencia negativa en el en el análisis de la esencia de la experiencia hermenéutica. ${ }^{7}$ Para Gadamer la tradición es el conjunto discursivo inseparable del acto de comprensión. Por ello nuestro filósofo menciona que la tradición es un verdadero compañero de comunicación, al que estamos vinculados como lo está el yo al tú. De esta forma, la relación que tenemos con el conjunto discursivo recibido por la historia es similar al que poseemos con otros individuos. Este tipo de vinculatividad es lo que Gadamer llama «vinculatividad moral» y argumenta que la experiencia hermenéutica es un proceso en el que interviene íntimamente la tradición; aunque esta última no es un simple objeto de conocimiento sino que es lenguaje, posee un comportamiento similar a un dialogante que habla por sí mismo.

Este pasaje al fenómeno moral de la hermenéutica es uno de los más importantes dentro del estudio gadameriano. Introduciéndose en él advertimos que Gadamer debe establecer una diferencia concluyente entre percibir la tradición como objeto y como un tú para no recaer en la hermenéutica regida bajo un método similar al de las ciencias. Por ello, diferencia dos tipos de experiencia del tú. La primera, como una experiencia donde el mismo es tomado como una experiencia general y se percibe al tú como un objeto más de mi mundo y no es tenido como persona sino, por el contrario, es medio y siempre en referencia al sí mismo, a la mismidad del yo. No se reconoce al otro como alter ego, como un otro yo que habla y se comporta con respecto a uno. En realidad, no hay lugar para la diferencia y todo se reduce a la mismidad del yo que interactúa con el otro como medio al igual que otro objeto a la mano que se domina. Esta forma de comprender la tradición se reduce a un tratamiento de la misma de forma metodológica en que la tradición enmudece y se subyuga a la dicotomía sujeto-objeto. Por el contrario, la experiencia del tú como fenómeno moral conlleva el reconocimiento del otro como alteridad reflexiva que demanda un condicionamiento del saber y el límite de las aspiraciones del sí mismo. Para el caso de la tradición debemos reconocer su instancia constitutiva en la interpretación. La conciencia histórica tiene la particularidad de validar tanto la alteridad constitutiva de nuestra propia historicidad como la historicidad de los otros. Esta 
vinculatividad esencial a la comprensión es una «vinculatividad moral» en la medida en que se reconoce la legitimidad de las pretensiones del otro.

La profundidad del razonamiento gadameriano no busca más que determinar estos diferentes modos de relación con la alteridad, en la cual las posibles consecuencias de pretensiones de dominio en la relación llevarían a una ruptura dialogal y a un no reconocimiento de sus pretensiones. Para este filósofo lo resultante del trato con la alteridad no puede nunca concebirse como objetivación ya que, en ese caso, se destruiría la vinculatividad moral. Esta exige experimentar al tú como realmente un tú, esto es, dejarse interpelar por él. Por ello, «la apertura hacia el otro implica, pues, el reconocimiento de que debo estar dispuesto a dejar valer en mí algo contra mí, aunque no haya ningún otro que lo vaya a hacer valer contra mí». ${ }^{8} \mathrm{La}$ apertura es ese «estar dispuesto a» que hace del otro un tú que se comporta con respecto a mí. Ésta, como ese estar dispuesto a dejar valer algo contra mí, se estructura sobre un mandato ético en última instancia. El diálogo que mantenemos con otros intérpretes supone siempre la disposición a considerar opiniones diversas a las nuestras como legítimas y las propias como relativas a nuestra historicidad; esto es, debemos ser capaces de reconocer la contingencia de la validez de nuestras opiniones. Esto es lo que Gadamer entiende por verdadera «experiencia hermenéutica». De hecho, la experiencia hermenéutica llega en verdad tan lejos como llegue la disposición al diálogo entre los seres racionales.

El punto radica, entonces, en que la obra debido a su carácter temporal acontece y cobra significado en la fusión de horizontes. El sentido, de esta forma, es inherente al texto mismo y no se sustenta ni en el autor ni el lector de la obra. Sin duda que el lector articula la comprensión desde su temporalidad pero esto no implica que sea una mera construcción de éste el texto. El propio carácter ontológico de la historia efectual hace que no pueda rebasar su finitud e iluminar por completo el conjunto de prejuicios. Siempre se halla un resto, un residuo inmanente que la distancia temporal no hace efectivo. Es éste el carácter infinito de la tarea hermenéutica de la comprensión en el que el lenguaje no se agota en la conciencia del intérprete ni tampoco en la conversación. Félix Duque dice: «comprender es inscribirse en un movimiento que viene de lejos, imprimiendo mutaciones tales en él que lo hagan reconocible como una pléyade de envíos».9 La noción de texto, por otro lado, viene a demarcar el límite de la tarea del lector. El «texto» para Gadamer es un concepto originalmente hermenéutico que

8 «Offenheit für den anderen schiesst also die Anerkennung ein, dass ich in mir etwas gegen mich gelten lassen muss, auch wenn es keinen anderen gäbe, der es gegen mich geltend machte». WM: 343 (VM: 438).

9 F. Duque. En torno al humanismo. Heidegger, Gadamer, Sloterdijk. Madrid, Tecnos, 2002, p. 109. 
tiene la función de ser el dato de autoridad en el fenómeno de la comprensión. Autoridad, que por otro lado, es dada por la apertura (Offenheit) del propio lector. El texto, en resumidas cuentas, es el «punto hermenéutico de identidad que limita todas las variables». ${ }^{10}$ Demarcación identitaria que no implica el agotamiento del mismo en una única lectura válida sino que representa el límite divisorio entre una interpretación ajustada al texto y una que excede los límites impuesto por él. Sobre ello resulta necesario mencionar la noción de «autonomía de sentido» (Sinnautonomie). ${ }^{11}$ Ésta tiene la pretensión de representar la idea de comunicación de una posible interpretación «objetiva» del texto guiado por ciertas normas internas de la estructura del mismo. Siendo, de esta forma, el sentido un conjunto semántico-pragmático que se desprende de la intención del autor y toma nuevas significaciones en el horizonte del lector.

Justamente por ello en este alcance gadameriano del círculo hermenéutico se halla un criterio de validez que permite articular la comprensión. Toda interpretación debe tener en cada proyección suya sobre el texto una coherencia con el sentido total del mismo. Esto lo habíamos mencionado anteriormente con el concepto de «anticipo de perfección». La articulación del sentido es una correspondencia entre el horizonte del intérprete instaurado en el presente y el horizonte de sentido de la obra enmarcada en el pasado. Es esto mismo lo que lleva a Martin Kusch a hablar de un «método» propio de la hermenéutica gadameriana. ${ }^{12}$ En el análisis que este autor hace de la fusión de horizontes observa tres rasgos característicos de ésta que se derivan de la noción de «horizonte» que Gadamer toma de Husserl. El primero que menciona es que Gadamer con la idea de fusión no hace más que devolver a primer plano el esquema moderno de sujeto-objeto. Esto mismo es lo que Heidegger le critica y lo que considera una crucial desviación de la hermenéutica ontológica de Sein und Zeit. Para este último esto representa una vuelta al sentido fenomenológico husserliano de «conciencia». Lo segundo que remarca Kusch es, justamente, que esta vuelta gadameriana al esquema moderno de conocimiento tiene la particularidad de darse de una forma dialogal. El hecho de que el sentido del texto sea un acontecimiento entre la fusión de horizontes de la obra y el intérprete habla de una vinculatividad dialógica fundamental a la hora de considerar la verdad del

$10 \ll[\ldots]$ Der Begriff «Text» ein ursprünglicher hermeneutischer Begriff ist. Er formuliert die autoritative Gegenbenheit, an der sich Verstehen und Auslegen zu messen hat - gleichsam als der hermeneutische Identitätspunkt, der alles Variable begrenzt». H. G. Gadamer. Ästhetik und Poetik I. Tubinga, Mohr, 1993, Band 8, p. 289 (trad. castellana Arte y verdad de la palabra, traducido por José Zuñiga García, Barcelona, Paidós, 1998, p. 100).

11 Cfr. WM: 372 (VM: 473).

12 M. Kusch. Language as calculus vs. language as universal medium. A study in Husserl, Heidegger and Gadamer. Dordrecht, Kluwer Academic Publishers, 1989, pp. 236-240. 
texto. ${ }^{13}$ El último punto que sugiere es el carácter crítico de la misma fusión de horizontes. Sobre ello cabe mencionar el mismo prólogo a la segunda edición de Wahrheit und Methode donde Gadamer aclara que la fusión de horizontes no implica una apropiación de la tradición de forma acrítica: «Si forma parte de la esencia de la tradición el que sólo exista en cuanto quien se la apropie, entonces forma parte seguramente de la esencia del hombre poder romper, criticar y deshacer la tradición». ${ }^{14}$

Según Kusch tenemos, entonces, que en el mismo acontecimiento interpretativo surge el método propio hermenéutico y éste posee una estructura dialógica. La visión crítica de la propia fusión alude a desenmascarar la visión común que se tiene de Gadamer como un mero conservador que valida de forma concluyente lo transmitido por la tradición. Esto no hace justicia a la posición gadameriana de la fusión de horizontes. Por el contrario, la hermenéutica filosófica propone que el acontecimiento propio del sentido es una conformación temporal que posee la característica de transformar ambas partes. Como dice Jean Grondin las partes de la fusión se «metamorfosean» (métamorphosent) engendrando una nueva obra. ${ }^{15} \mathrm{La}$ fusión de horizontes lo que hace es transformar mediante un evento temporal el horizonte de sentido sus elementos constituyentes, si se quiere del sujeto comprensor y el objeto comprendido, creando una nueva obra. De esta manera Grondin alude a dos momentos esenciales de la comprensión. El primer momento propio de la fusión en el que el ente comprensor se fusiona con lo comprendido; $\mathrm{y}$, el segundo momento, donde debido a lo anterior se ilumina una nueva concepción del texto. Un ejemplo claro de esto lo podemos encontrar en la lectura de los clásicos, que tanto preocupó a Gadamer. La distancia temporal que actúa en la configuración del sentido cobra nuevas dimensiones cada vez que una época lo lee.

Un caso ejemplar de ello son los tratados de Aristóteles. Hoy en día podemos confrontar, si esto nos es permitido, la interpretación de Tomás de Aquino y la de Heidegger. Para Gadamer la obra aristotélica marca el límite y el punto identitario de las interpretaciones que se actualiza en los momentos históricos de la lectura. Lo que tenemos con estos dos intérpretes es, según la distancia temporal que cada uno tiene con la obra, un acontecimiento del sentido donde los propios tratados aristotélicos cobran nuevo valor y ellos mismos se ven afectados por el discurso

13 Kusch menciona lo siguiente: «the hermeneutical dialogue is in fact a description of a method for obtaining the truth». Kusch, M. Language as calculus vs. language as universal medium, op., cit, p. 238.

14 «Mag es immerhin zum Wesen der Tradition gehören, nur durch Aneignung zu sein, so gehört es doch gewiss auch zum Wesen des Meschen, Tradition brechen, kritisieren und auflösen zu können». WM: XXV (VM: 20).

15 Cfr. J. Grondin. «La fusion des horizons. La version gadamérienne de l'adaequatio rei et intellectus?» en Archives de philosophie, 68, 2005, pp. 401-418. 
comprendido. La fusión horizóntica de los intérpretes y la obra, como vimos recién, no sólo metamorfosea las partes sino también ilumina la obra con una nueva configuración significativa. Tal es así que hablamos tanto de una lectura tomista de Aristóteles como de una lectura heideggeriana de Aristóteles, para el caso. Ahora bien, esto no implica ni que el sentido sea producido totalmente por los intérpretes ni que una interpretación sea «más» valida que la otra. La autonomía propia del sentido de la obra destaca los límites interpretativos. Lo que tenemos, en realidad, con cada comprensión es el desarrollo de las posibilidades inherentes del sentido. Que pueda haber tanto una lectura tomista como heideggeriana de Aristóteles indica que la obra de éste último tiene la posibilidad interna de responder a ciertos cuestionamientos de cada época.

Es esto mismo lo que Gadamer entiende por «clásico». La riqueza del texto radica, justamente, en que pueda responder ante requerimientos de distintas épocas. El sentido no es más que la continuidad de las acepciones que se van induciendo unas a otras a lo largo del tiempo. Esto no implica un relativismo interpretativo; no es que existe un sentido en sí incognoscible donde la interpretación correcta se acercaría al mismo. No hay un acercamiento infinitesimal a un supuesto «en sí» del texto. El sentido se conforma a través del tiempo según las distintas acepciones que recibe. Él es las distintas acepciones realizadas. Pero, y este es el punto que nos interesa resaltar a nosotros, las diferentes interpretaciones no agotan las posibilidades realizativas del texto. La lectura tomista, podemos decir, fue «adecuada» a su tiempo mientras que la de Heidegger también incluso pero esto no implica que los tratados aristotélicos no respondan otras inquietudes diversas en el futuro. Siempre nos hallamos ante un residuo semántico que convierte a la tarea hermenéutica en una tarea infinita. De allí que la hermenéutica gadameriana haya sido catalogada como una semántica inefable. ${ }^{16} \mathrm{Y}$ esto mismo es lo que nosotros entendemos por la indisponibilidad del lenguaje en la interpretación. La relación semántica entre la palabra y la cosa siempre posee un fondo inalcanzable, inefable. Gadamer dice: «Todo hablar humano es finito en el sentido de que en él yace la infinitud de un sentido por desplegar e interpretar». ${ }^{17}$

\section{EL CARÁCTER AMBIGUO DE LA FUSIÓN DE HORIZONTES}

Como hemos observado la noción de sentido verdadero conlleva en el planteamiento gadameriano dos características fundamentales. Por un lado muestra

16 Kusch, M. Language as calculus vs. language as universal medium, op. cit, p. 256.

17 «Alles menschliche Sprechen ist in der Weise endlich, dass eine Unendlichkeit des auszufaltenden und auszulegenden Sinnes in ihm angelegt ist». WM: 434 (VM: 549). 
que el sentido es un constructo de la fusión de horizontes y; por otro lado, que el mismo siempre se mantiene autónomo y, por tanto, inefable. Estas dos particularidades esenciales se corresponden con el análisis que realiza Gadamer del concepto de «mundo en sí» (Welt an sich) en uno de los últimos apartados de Wahrheit und Methode. ${ }^{18}$ Podemos decir que en este tratamiento del «mundo en sí» Gadamer desarrolla su punto de vista hermenéutico con respecto al problema de la comprensión a un nivel multicultural. La pregunta guía para el caso será «¿cómo es posible la fusión de horizontes para esquemas conceptúales distintos?». ¿La fusión de horizontes posee el mismo comportamiento frente al caso de interpretaciones divergentes culturalmente que frente a la interpretación de textos? ¿La característica de la autonomía de sentido junto a una semántica inefable funciona de igual manera en el diálogo intercultural?

Gadamer, como hemos venido observando, confiere al diálogo un valor de importancia a la hora de encontrar la palabra «justa» que explicite el sentido de un discurso. Para el caso, anteriormente habíamos observado junto a Kusch que el «método» propio de esta hermenéutica filosófica es el diálogo. No en vano es éste el camino y el modo de acceso propio al objeto de investigación. Esto se puede observar en la explicación de la importancia del lenguaje a la hora de explicitar el ente. ${ }^{19}$ Gadamer se encarga de aclarar que el lenguaje es el modo que tiene el hombre de elevarse por sobre su entorno (Umwelt). En una especie de antropología hermenéutica distingue al hombre de animal a partir de la capacidad de aquel de simbolizar el mundo (Welt) mediante el lenguaje. La capacidad lingüística que posee el hombre es lo que lo aleja del animal. Retornando a Aristóteles Gadamer continúa con la diferencia entre animal y hombre a partir de la distinción entre el simple sonido de la voz, de los animales, y la voz acompañada de significado, de los hombres. Esta diferencia aristotélica le permite a nuestro autor establecer la identidad a priori de mundo y lenguaje. Que el hombre posea lenguaje implica que éste pueda elevarse de su entorno. Eso mismo es lo que lleva, a su vez, a la determinación antropológica de que el hombre posee la capacidad de alzarse de su entorno. Ello no implica que abandone su entorno sino que tiene una posición completamente distinta del animal. La «elevación» del hombre se entiende a partir de la libertad intrínseca que tiene de nombrar su mundo. Posee la libertad de ejercicio variado de su propia capacidad lingüística de nombrar y articular un discurso sobre su entorno. En el lenguaje del hombre, la voz acompañada de significado, habla el mundo. El hombre tiene mundo porque posee lenguaje y no a la inversa, y la libertad de su ejercicio de nombrar es lo que nos lleva a una multiplicidad diciente sobre el mundo. Esta genealogía de la lingüisticidad cultural del hombre lleva a enunciar dos proposiciones fundamentales. 
1. La primera es que al mundo se accede mediante el lenguaje. El ente no es el objeto que se encuentra en el enunciado sino que accede a los enunciados mediante la capacidad de articular un discurso con sentido del hombre. Esto es algo que Gadamer ha resumido en su célebre frase «el ser que puede ser comprendido es lenguaje».

2. La segunda es que el lenguaje se funda en la conversación y, a partir de ello, que la lingüisticidad es potencialmente abarcativa de otras formas de comprensión del mundo.

La explicitación de esta segunda tesis que extraemos de la argumentación gadameriana se ofrece también a partir de un análisis aristotélico sobre el lenguaje. Gadamer recurre a Aristóteles para mostrar que el lenguaje tiene su ser en la conversación. El punto de referencia es el pasaje de la Política 1253 a 10 en que el estagirita describe que la posibilidad de entenderse mutuamente se debe a un acuerdo previo de la comunidad hablante. Además de diferenciarse el hombre del animal según la distinción entre la simple voz y la voz dotada de significado Aristóteles, a partir de la lectura gadameriana, dictamina que la designación de las palabras no se da por naturaleza sino por convención (symbolon). Esta consideración convencional del lenguaje radica en el consenso de una comunidad sobre lo que considera bueno y correcto. La validez del uso de las palabras se encuentra a partir de la noción de nomoi; aquello que los hombres consideran para sí productivo a la hora de alcanzar la comprensión mutua. De este modo, en Gadamer, el ámbito semántico del lenguaje tiene por origen el ámbito pragmático del mismo. Con esto se descubre la esencia del lenguaje como la realización comunicativa de la lengua y, por otro lado, se observa que la lingüisticidad se ofrece como el horizonte de sentido de toda posible experiencia del mundo. Justamente, Gadamer menciona que «el mundo lingüístico propio en el que se vive no es una barrera que impide todo conocimiento del ser en sí, sino que abarca por principio todo aquello hacia lo cual puede expandirse y elevarse nuestra percepción». ${ }^{20}$

A partir de este examen gadameriano, que en cierto término podemos suponer como una génesis del lenguaje, se establece que la lingüisticidad es potencialmente abarcativa de toda forma de comprensión; o sea, de toda acepción del lenguaje vertida en una lengua. Ya, para Gadamer, toda lengua es no sólo forma sino contenido de una acepción lingüística del mundo; diferenciándose de esta forma con la concepción de la relación entre lenguaje e historia de Humboldt. En ello reconocemos dos puntos cruciales. El primero es que toda lengua en sí misma es expresión del mundo. Segundo, debido a ello, toda decir de la lengua

20 «Die eigene Sprachwelt, in der einer lebt, ist nicht eine Schranke, die Erkenntnis des Ansichseins verhindert, sondern umfasst grundsätzlich alles, wohinein sich unsere Einsicht zu erweitern und zu erheben vermag». WM: 423 (VM: 536). 
posee como tal una pretensión de verdad. Toda lengua es una percepción del mundo. De forma similar a lo que anteriormente habíamos observado en el caso de los textos las distintas percepciones del mundo (las diferentes lenguas) constituyen las variadas interpretaciones surgidas a partir del «texto» que Gadamer, recobrando un viejo problema filosófico, llama «mundo en sí̀. Ahora bien, para nuestro filósofo esto no implica que las diferentes acepciones del mundo en cada lengua sean relativas a un mundo en sí incognoscible. Plantear de semejante forma la alteridad de las interpretaciones existentes sería postular, en definitiva, un punto de vista teológico o incluso solipsista. No podemos pensar en horizontes cerrados que de un modo etnocentrista intentan alcanzar la cosa en sí de una manera excluyente. Inevitablemente esto lleva a pensar su lengua como «la» lengua del mundo. El procedimiento que encuentra Gadamer de no caer en esta problemática es de igual forma que la desarrollada en la interpretación de textos. Si más arriba habíamos mencionado la noción de «autonomía de sentido» como la delimitación y, a su vez, como condición de posibilidad de diversas configuraciones de sentido ahora diremos que la noción de «mundo en sí» actúa análogamente y que, por tanto, el mundo no es más que las diferentes acepciones que tenemos de él. Así como las multiplicidades interpretativas de un texto se configuran a partir del desarrollo de la capacidad que tiene de responder a diferentes cuestionamientos el «mundo en sí» es la conformación de los distintos alcances lingüísticos que resultan de su experiencia.

Y es esto mismo lo que lleva a Brice Wachterhauser a definir a Gadamer de «realista metafísico». ${ }^{21}$ Wachterhauser interpreta que, en este punto, la hermenéutica gadameriana muestra su fiel adhesión al programa platónico de la concepción de las ideas. Aunque el «mundo en sí» no sea más que el conjunto de acepciones del mundo esto implico por sí mismo que el «mundo en sí» es inteligible. El reconocimiento de la inteligibilidad del mundo como presupuesto es lo que lleva a que las diferentas lenguas puedan hacerlo presente en sus lenguas particulares. A pesar de que la realidad del mundo no depende de nuestro punto de vista sí se hace inteligible desde nuestro lugar. Esto conlleva dos consecuencias esenciales. La primera es que en el fondo la hermenéutica filosófica no es un relativismo; y la segunda, que tampoco su planteamiento puede ser tildado de «idealismo lingüístico». Gadamer no es un escéptico relativista. Es un realista metafísico porque el mundo es inherentemente inteligible. La experiencia del mundo es discursiva porque, justamente, el mundo es perceptible en sí mismo. ${ }^{22}$

21 B. Wachterhauser. «Getting it Right: Relativism, Realism, and Truth» en R. Dostal. (ed.) The Cambrige Companion to Gadamer, Cambridge, Cambridge University Press, 2002, pp 52-78.

22 «Instead our experience can mediate between language and the world because all three are in principle "intelligible"». Ibid. p. 77. 
De esta forma, Gadamer no sólo plantea la relación de identidad entre pensamiento y lenguaje sino también la forma de no caer en un etnocentrismo. Para este autor la diversidad lingüística no significa un perspectivismo excluyente sino, por el contrario, incluyente. La lingüisticidad, por tanto, es potencialmente abarcativa de todo discurso sobre el mundo. La multiplicidad de lenguas no significa un relativismo del mundo sino que éste es las distintas acepciones en que se ofrece, como hemos observado. Esto nos lleva a considerar la posibilidad de correspondencia que existe entre la postura de Gadamer y Taylor con respecto al carácter ontológico del lenguaje. Tanto para uno como para otro el concepto de «historia» se mantiene en la noción aristotélica de «potencia» (dynamis). Para Taylor «Hablar de potencialidades no significa suponer un conjunto unitario. Podemos reconocer $-\mathrm{y}$, de hecho, lo hacemos cada vez más- diversas líneas de desarrollo posible, algunas de las cuales parecen mutuamente incompatibles, al menos a primera vista». ${ }^{23}$ Podemos decir que Gadamer adhiere a este pensamiento en cuanto el sentido no representa una univocidad sino acontecimientos epocales que se desprenden de sus propias posibilidades interpretativas. Esto significa reconocer en la postura gadameriana una visión sincrónica y diacrónica de la fusión de horizontes. Ésta última, de hecho, opera en ambas direcciones. La génesis lingüística se estructura históricamente según una vía interpretativa que conforma una tradición a partir de la cual cada cultura se comporta ante el mundo. Contra el monopolio y unilateralidad de la historia (Geschichte) planteada en la noción del despliegue del espíritu (Geist) en Hegel el concepto aristotélico de «potencia» nos brinda la posibilidad de pensar la historia como diversos encauces o acepciones que no son excluibles mutuamente. Que la historia sea explicable desde la idea aristotélica de potencia y que la lingüisticidad sea, a su vez, potencialmente abarcativa no es casualidad. Ambas se corresponden en la comprensión y entendimiento del hombre con el mundo y sus semejantes. Justamente allí se inscribe la fusión de horizontes como el elemento hermenéutico relevante a la hora de lograr un entendimiento en común entre las diferentes líneas históricas y lingüísticas.

El fin de la fusión de horizontes no es más que lograr un acuerdo lingüístico sobre la base de un lenguaje común. Junto a Grondin dijimos que la fusión posee dos características fundamentales. Por un lado la transformación de las partes fusionadas y; por otro lado, la conformación de un nuevo sentido. Para el caso, las partes fusionadas en tanto son tradiciones o conjuntos discursivos cambian sus respectivos horizontes ampliándolos y logran formar un lenguaje en común

23 «To speak of potentialities doesn't mean to suppose a unitary set. We can and increasingly do recognize diverse lines of possible development, some of which seem incompatible with each other, at least a first blush». Ch. Taylor. «Comparison, History, Truth», op. cit., p. 162 (p. 217). 
por el cual consiguen entenderse. Ahora bien, todo ello parece contradecirse si prestamos atención a una problemática interna de la fusión de horizontes. Algo que precisamente Charles Taylor no menciona.

La fusión de horizontes sólo es posible en cuanto la dialéctica de la pregunta y repuesta es puesta práctica en la forma de que nos dejemos interpelar por la alteridad y su conjunto discursivo con pretensión de verdad. Por eso habíamos mencionado que la fusión no puede darse si no es sobre la base de la «vinculatividad moral» del diálogo hermenéutico. La realización de la fusión se ofrece como la dialéctica de la alteridad. Pero, para Gadamer, dicha dialéctica de la alteridad queda subordinada al desarrollo de la dialéctica de la cosa (Sache) expresada en la conversación. De allí que en este autor aparezca la metáfora del «juego» como la descripción del movimiento mismo de la cosa en el pensar dialogal. Una noción que en Wahrheit und Methode había aparecido en su primera parte como descripción del movimiento comprensivo del arte y que en esta tercera y última parte reaparece como el elemento descriptivo de la enérgeia del lenguaje; lugar que por otro lado es el propio de la comprensión. Para Gadamer la idea suya de «juego lingüístico» se corresponde con su idea del acontecimiento dialogal de la tradición en el lenguaje. El devenir de la conversación entre una y otra comunidad lingüística, de esta manera, queda supeditado al devenir propio de la tradición en la que me encuentro. Por eso Gadamer nos dice que el verdadero subjectum del diálogo, del juego lingüístico, es el acontecer de la cosa y no los intérpretes que intervienen en él. Ante ello explica que: «Tampoco aquí puede hablarse de un jugar con el lenguaje o con los contenidos de la experiencia del mundo o de la tradición que nos hablan, sino de un juego del lenguaje mismo que nos habla, que propone y se retira, que pregunta y que se cumple a sí mismo en la respuesta». ${ }^{24}$

Con ello tenemos un pensar metafísico que parece derrumbar la tesis hermenéutica del reconocimiento de la alteridad como hemos venido observando hasta ahora ya que la fusión de horizontes no es más que el juego mismo que la tradición juega más allá de sus intérpretes e intervenciones de esquemas conceptuales distintos. Parece, en realidad, que el lenguaje común como una nueva iluminación en el comparecer de la experiencia hermenéutica se reduce tan sólo a la transformación de un único horizonte. La alteridad viene a cumplirse como un simple medio de comprensión del sí mismo. La alteridad es apariencia de un juego lingüístico encerrado en él mismo. Y con ello tenemos más bien un acercamiento al pensar histórico de Hegel que un alejamiento de él. La alteridad

24 «Entsprechend ist auch hier nicht von einem Spielen mit der Sprachen oder mit den und ansprechenden Inhalten der Welterfahrung oder Überlieferung die Rede, sondern von dem Spiel der Sprache selbst, die uns anspricht, vorschlägt und zurïckzieht, fragt und in der Antwort sich selbst erfüllt». WM: 464 (VM: 584). 
de otro horizonte histórico es sólo la proyección y continuidad a través de una transformación de un horizonte único que mantiene su identidad inalterada. Esta parece ser la problemática mayor que encontramos en el desarrollo del pensamiento hermenéutico de Gadamer en Wahrheit und Methode. Y, debido a la explícita influencia de Gadamer en Taylor, parece que este problema también le incumbe al filósofo canadiense.

\section{CONCLUSIONES}

En los apartados anteriores hemos intentado analizar en profundidad la funcionalidad de la fusión de horizontes en la propuesta hermenéutica gadameriana con respecto a la problemática suscitada a partir del citado trabajo de Charles Taylor. Un interés particular nos llevó a ello: la clara referencia del trabajo de Taylor a Gadamer. La respuesta ante la posibilidad de lograr una comprensión intercultural es la fusión de horizontes gadameriana con las características vistas. Pero, ante ello, no podemos dejar de observar la problemática interna que encuentra en la argumentación de Wahrheit und Methode. La forma racional que busca el autor de Argumentos filosóficos de obtener una comprensión de culturas diversas a la propia se basa en la resignificación de la noción de «historia» de origen moderno. Ante la idea de una sola línea de desarrollo histórico propone diversas líneas posibles. Según ello existiría la posibilidad de comparar los esquemas discursivos divergentes sin que se produzca ninguna distorsión y, de esta forma, alcanzar una universidad lingüística fáctica. La comparación de lenguajes como formas de vida busca el reconocimiento de la alteridad sin ningún tipo de intervención dogmática de una cultura en otra. La distorsión, a nuestro parecer, sí la encontramos en la ambigüedad de la argumentación gadameriana en torno a la fusión de horizontes. O bien estamos hablando de la fusión de horizontes distintos o bien estamos hablando de la transformación de un único horizonte de comprensión. Taylor no da respuesta a esta problemática hermenéutica a pesar de tomar a Gadamer como principal referente. Quizás el punto radique en que la fusión de horizontes que plantea sea restrictiva con respecto a las consecuencias de la metáfora gadameriana de juegos lingüísticos. En Taylor no encontramos una subordinación de los sujetos hablantes a una lingüisticidad omniabarcante potencialmente abierta a toda comprensión del mundo. En el «proyecto comparativista» las subjetividades culturales deciden y despliegan sus horizontes más allá o más acá, como se lo quiera ver, de una perspectiva céntrica del lenguaje. Podemos decir que el planteamiento comparativista elude o restringe el elemento metafísico de la fusión de horizontes tal cual es presentada por Hans-Georg Gadamer en Wahrheit und Methode. Este es un intento de respuesta y explicación de una omisión que lejos de ser 
inocente adquiere un valor fundamental a la hora de responder al problema de comprensión de culturas ajenas a la nuestra.

\section{REFERENCIAS BIBLIOGRÁFICAS}

DUQUE, F. 2002: En torno al humanismo. Heidegger, Gadamer, Sloterdijk. Madrid, Tecnos.

GADAMER, H-G. 1993: Ästhetik und Poetik I. Tubinga, Mohr, Band 8 (trad. castellana Arte y verdad de la palabra, traducido por José Zuñiga García, Barcelona, Paidós, 1998).

GADAMER, H-G. 1975: Wahrheit und Methode. Mohr, Tubinga (trad. castellana Verdad y Método, traducción por Ana Aparicio-Rafael de Agapito, Salamanca, Sígueme, 1977).

GRONDIN, J. 2005: «La fusion des horizons. La version gadamérienne de l'adaequatio rei et intellectus?» en Archives de philosophie, 68, pp. 401-418.

HEIDEGGER, M. 1963: Sein und Zeit. Max Niemayer, Tubinga (trad. castellana Ser y Tiempo, traducción por Jorge Rivera, Santiago de Chile, Editorial Universitaria, 1997).

KUSCH, M. 1989: Language as calculus vs. language as universal medium. A study in Husserl, Heidegger and Gadamer. Dordrecht, Kluwer Academic Publishers.

TAYLOR, Ch. 1995: «Comparison, History, Truth» en Taylor, Ch. Philosophical Arguments, Cambridge-London, Harvard University Press (trad. castellana Taylor Ch. Argumentos filosóficos. trad. Fina Birulés Bertrán, Barcelona, Paidós, 1997).

WACHTERHAUSER, B. 2002: «Getting it Right: Relativism, Realism, and Truth» en Dostal, R. (ed.) The Cambrige Companion to Gadamer, Cambridge, Cambridge University Press. 
El carácter antbiguo de la fusión de horizontes... 